This item was submitted to Loughborough's Research Repository by the author.

Items in Figshare are protected by copyright, with all rights reserved, unless otherwise indicated.

\title{
Lag synchronization of switched neural networks via neural activation function and applications in image encryption
}

PLEASE CITE THE PUBLISHED VERSION

http://dx.doi.org/10.1109/TNNLS.2014.2387355

\section{PUBLISHER}

(c) Institute of Electrical and Electronics Engineers

\section{VERSION}

AM (Accepted Manuscript)

\section{PUBLISHER STATEMENT}

This work is made available according to the conditions of the Creative Commons Attribution-NonCommercialNoDerivatives 4.0 International (CC BY-NC-ND 4.0) licence. Full details of this licence are available at: https://creativecommons.org/licenses/by-nc-nd/4.0/

\section{LICENCE}

CC BY-NC-ND 4.0

\section{REPOSITORY RECORD}

Wen, Shiping, Zhigang Zeng, Tingwen Huang, Qinggang Meng, and Wei Yao. 2015. "Lag Synchronization of Switched Neural Networks via Neural Activation Function and Applications in Image Encryption". Loughborough University. https://hdl.handle.net/2134/17204. 


\title{
Lag Synchronization of Switched Neural Networks via Neural Activation Function and Applications in Image Encryption
}

\author{
Shiping Wen, Zhigang Zeng, Senior Member, IEEE, Tingwen Huang, Senior Member, IEEE, \\ Qinggang Meng, and Wei Yao
}

\begin{abstract}
This paper investigates the problem of global exponential lag synchronization of a class of switched neural networks with time-varying delays via neural activation function and applications in image encryption. The controller is dependent on the output of the system in the case of packed circuits, since it is hard to measure the inner state of the circuits. Thus, it is critical to design the controller based on the neuron activation function. Comparing the results, in this paper, with the existing ones shows that we improve and generalize the results derived in the previous literature. Several examples are also given to illustrate the effectiveness and potential applications in image encryption.
\end{abstract}

Index Terms-Exponential stability, lag synchronization, switched neural networks.

\section{INTRODUCTION}

$\mathbf{H}$ YBRID systems have been investigated extensively with the rapid development of intelligent control. As a special case of hybrid systems, switched systems consist of a family subsystems, which are controlled by a switching rule. In reality, many systems can be modeled as switched systems, switched circuits, switched networks, and so on. Considerable attention has been drawn to the theoretical analysis of switched systems [1].

Meanwhile, synchronization of neural networks has attracted great attention due to its potential applications in many fields such as secure communications, biological systems, information science, image encryption,

Manuscript received August 12, 2014; revised October 21, 2014 and December 21, 2014; accepted December 27, 2014. This work was supported in part by the Qatar National Research Fund, a member of the Qatar Foundation through the National Priorities Research Programme under Grant 4-1162-1-181, in part by the Program for the Changjiang Scholars and Innovative Research Team in the University of China under Grant IRT1245, in part by the National Natural Science Foundation of China under Grant 61125303, Grant 61203286, and Grant 61403152, and in part by the National Basic Research Program (973 Program) of China under Grant 2011CB710606.

S. Wen and Z. Zeng are with the School of Automation, Huazhong University of Science and Technology, China and the Key Laboratory of Image Processing and Intelligent Control, Ministry of Education, Wuhan? 430074, China.

T. Huang is with Texas A\&M University at Qatar, Doha 23874, Qatar (e-mail: tingwen.huang@qatar.tamu.edu).

Q. Meng is with the Department of Computer Science, Loughborough University, Loughborough LE11 3TU, U.K. (e-mail: q.meng@lboro.ac.uk).

W. Yao is with the School of Computer Science, South-Central University for Nationalities, Wuhan 430074, China (e-mail: hevigreen@gmai.com).
0

]

0 pseudorandom number generator, and adaptive dynamic programmer [2]-[14]. Synchronization phenomena including complete synchronization [15]-[17], generalized synchronization [18], phase synchronization [19], and lag synchronization [20] have been investigated. In connected electronic networks, time delays are unavoidable due to finite signal transmission times, switching speeds, and the complete synchronization of neural networks with time delays hard to implement effectively, but we can implement lag synchronization.

Several control methods have been proposed for the lag synchronization of delayed neural networks, such as periodically intermittent control in [21]-[23]. Exponential stability criteria are derived for the synchronization error systems with constant time delays in [21] and [22], however, these criteria are not applicable for systems with time-varying delays. Meanwhile, only asymptotical stability criteria are derived for synchronization error systems in [23]. Li and Bohnerb [24] investigated the exponential synchronization of chaotic neural networks via linear matrix inequality techniques. However, there are a few results on global exponential lag synchronization of switched neural networks.

Motivated by the above discussion, in this paper, we investigate the problem of globally exponential lag synchronization for a class of switched neural networks with time-varying delays. It is worth pointing out that, the proposed problem is nontrivial because of the difficulties such as the controller is designed via the neuron activation function.

The rest of this paper is organized as follows. In Section II, preliminaries are given. In Section III, a new model of synchronization error system is formulated within a unified framework. In Section IV, synchronization of switched neural networks is discussed by the controller based on the neuron activation function. Several sufficient conditions are derived to ensure the synchronization of switched neural networks. Analysis has been made on results in this paper and the previous ones. In Section V, two illustrative examples are discussed to demonstrate the effectiveness of the theoretical analysis. Finally, the conclusion is drawn in Section VI.

\section{PRELIMINARIES}

Denote $u=\left(u_{1}, \ldots, u_{n}\right)^{T},|u|$ as the absolute-value vector, i.e., $|u|=\left(\left|u_{1}\right|,\left|u_{2}\right|, \ldots,\left|u_{n}\right|\right)^{T},\|x\|_{p}$ as the $p$-norm of vector $x, 1 \leq p<\infty$. $\|x\|_{\infty}=\max _{i \in\{1,2, \ldots, n\}}\left|x_{i}\right|$ is the 


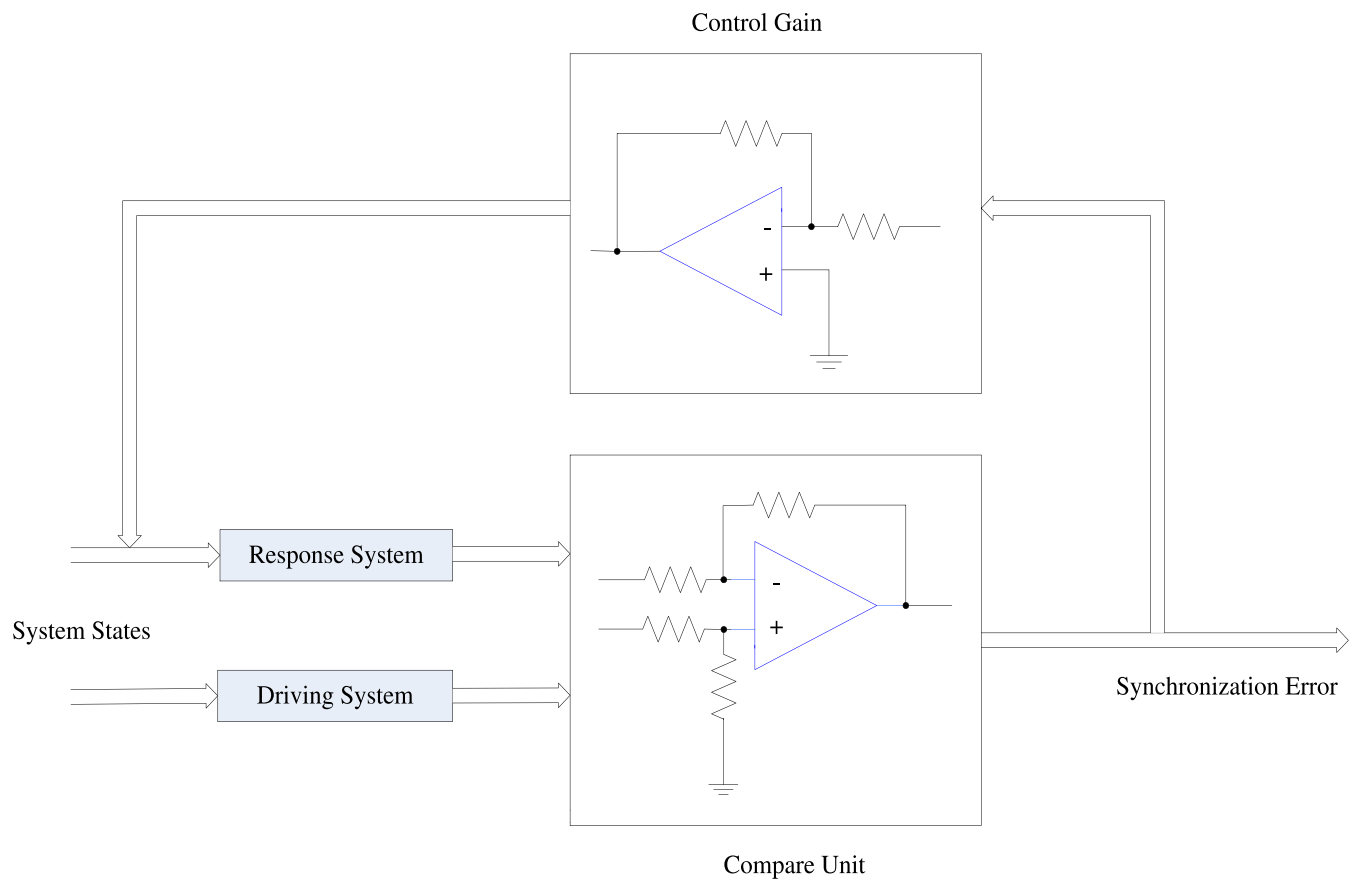

Fig. 1. Synchronization scheme of coupled memristive neural networks.

infinity norm of vector $x$. Denote $\|D\|_{p}$ as the $p$-norm of the matrix $D$. Denote $\mathcal{C}$ as the set of continuous functions.

A set of neural networks is considered as the individual subsystems of the switched neural network. The driving switched neural network is described as follows:

$$
\dot{x}(t)=-C_{l} x(t)+A_{l} f(x(t))+B_{l} f(x(t-\tau(t)))+I
$$

where $l$ is a switching signal taking its value in the finite set $\mathcal{I}=\{1, \ldots, N\}$, which means that the matrices $\left(A_{l}, B_{l}, C_{l}\right)$ are allowed to take values in the finite set $\left\{\left(A_{1}, B_{1}, C_{1}\right), \ldots,\left(A_{N}, B_{N}, C_{N}\right)\right\}$. The parameters of system (1) are utilized to reflect the switched property of the electronic elements in neural networks, such as switched resistors and so on.

Throughout this paper, we assume that the switching rule $l$ is known priori to the receiver and its instantaneous value is available in real time. The initial condition of system (1) is in the form of $x(t)=\phi(t) \in \mathcal{C}\left([-\chi, 0], \mathbb{R}^{n}\right), \chi=\max \{\bar{\tau}\}$, where $\bar{\tau}=\max _{1 \leq l \leq N} \bar{\tau}_{l}, 0 \leq \tau_{l}(t) \leq \bar{\tau}_{l}$.

Consider the following response system:

$$
\dot{x}(t)=-C_{l} x(t)+A_{l} f(x(t))+B_{l} f(x(t-\tau(t)))+I .
$$

Define an indicator function $\Pi(t)=\left(\Pi_{1}(t), \ldots, \Pi_{N}(t)\right)^{T}$, where

$$
\Pi_{l}(t)= \begin{cases}1, & \text { when the switched system is described } \\ & \text { by the } l \text { th mode }\left(A_{l}, B_{l}, C_{l}\right) \\ 0, & \text { otherwise }\end{cases}
$$

with $l=1, \ldots, N$. Then, the driving switched neural network (1) can be represented by

$$
\begin{aligned}
\dot{x}(t)=\sum_{l=1}^{N} \Pi_{l}(t)\left(-C_{l} x(t)\right. & +A_{l} f(x(t)) \\
+ & \left.B_{l} f(x(t-\tau(t)))+I\right) .
\end{aligned}
$$

It follows that $\sum_{l=1}^{N} \Pi_{l}(t)=1$ under any switching rules. Assume the response system has the same switching law as the driving system

$$
\begin{aligned}
\dot{y}(t)=\sum_{l=1}^{N} \Pi_{l}(t)(- & C_{l} y(t)+A_{l} f(y(t)) \\
& \left.+B_{l} f(y(t-\tau(t)))+I+u_{l}(t)\right)
\end{aligned}
$$

where $u_{l}(t)(l=1, \ldots, N)$ are the controllers. The initial condition of system (5) is in the form of $y(t)=\varphi(t) \in$ $\mathcal{C}\left([-\bar{\tau}, 0], \mathbb{R}^{n}\right)$.

The synchronization scheme of coupled switched neural networks can be presented as in Fig. 1, in which, we have the synchronization error state via the compare units and the control input.

In this paper, we assume the following.

A1: For $i \in\{1,2, \ldots, n\}$, the activation function $f_{i}$ is Lipchitz continuous; and $\forall r_{1}, r_{2} \in \mathbb{R}$, there exists real number $l_{i}$ such that

$$
0 \leq \frac{f_{i}\left(r_{1}\right)-f_{i}\left(r_{2}\right)}{r_{1}-r_{2}} \leq \iota_{i}
$$

A2: For $i \in\{1,2, \ldots, n\}, \tau_{i}(t)$ satisfies

$$
0 \leq \tau_{i}(t) \leq \bar{\tau}_{i}, \quad \dot{\tau}_{i}(t) \leq \mu_{i}<1 .
$$

To derive sufficient conditions for the global exponential lag synchronization of system (4) with system (5), we will need the following lemmas.

Lemma 1 [25]: Given any real matrices $X, Z, P$ of appropriate dimensions and a scalar $\varepsilon_{0}>0$, where $P>0$, the following inequality holds:

$$
X^{T} Z+Z^{T} X \leq \varepsilon_{0} X^{T} P X+\varepsilon_{0}^{-1} Z^{T} P^{-1} Z .
$$


In particular, if $X$ and $Z$ are vectors, $X^{T} Z \leq 1 / 2$ $\left(X^{T} X+Z^{T} Z\right)$.

\section{NEW MOdEL FOR THE SYNCHRONIZATION ERROR SYSTEM}

It is hard to obtain real-time inner states of the integrated and packed circuit, the output of this circuit can be utilized to measure such packed circuit. Therefore, we aim to design a controller of the circuit based on its output function, which is also called activation function in neuromorphic circuit, and to reach lag synchronization $[y(t) \rightarrow x(t-\xi)$ for some constant lag time $\xi>0$ ]. The error system can be obtained as

$$
\begin{aligned}
\dot{e}(t)=\sum_{l=1}^{N} \Pi_{l}(t)\left(-C_{l} e(t)\right. & +A_{l} \Phi(e(t)) \\
& \left.+B_{l} \Phi(e(t-\tau(t)))+u_{l}(t)\right)
\end{aligned}
$$

where $e(t)=\left(e_{1}(t), e_{2}(t), \ldots, e_{n}(t)\right)^{T}$ is the lagsynchronization error, and

$$
e_{i}(t)=y_{i}(t)-x_{i}\left(t-\xi_{i}\right) .
$$

The neural activation functions with/without delays are

$$
\begin{aligned}
\Phi(e(t))= & \left(\Phi_{1}\left(e_{1}(t)\right), \ldots, \Phi_{n}\left(e_{n}(t)\right)\right)^{\mathrm{T}} \\
= & f(e(t)+x(t-\xi))-f(x(t-\xi)) \\
\Phi(e(t-\tau(t)))= & \left(\Phi_{1}\left(e_{1}\left(t-\tau_{1}(t)\right)\right), \ldots, \Phi_{n}\left(e_{n}\left(t-\tau_{n}(t)\right)\right)\right)^{\mathrm{T}} \\
= & f(e(t-\tau(t))+x(t-\tau(t)-\xi)) \\
& -f(x(t-\tau(t)-\xi)) .
\end{aligned}
$$

In the case of packed circuits, it is hard to measure the inner state of the circuits, the controller is dependent on the output of the systems. An output controller is designed in this paper as follows:

$$
u_{l}(t)=K_{l} \Phi(e(t))
$$

where $K_{l}=\left(k_{l i j}\right)_{n \times n}$ is a constant gain matrix to be determined to synchronize the drive and response systems, and $\Phi(e(t))$ is the output function without delays.

With controller (8), the error system (6) is transformed into

$\dot{e}(t)=\sum_{l=1}^{N} \Pi_{l}(t)\left(-C_{l} e(t)+\hat{A}_{l} \Phi(e(t))+B_{l} \Phi(e(t-\tau(t)))\right)$

where $\hat{A}_{L}=\left(\hat{a}_{l i j}\right)_{n \times n}=\left(a_{l i j}+k_{l i j}\right)_{n \times n}$.

\section{MAin Results}

To gain the main results, the following lemma is introduced. The initial condition of system (9) is in the form of $e(t)=\varphi(t)-\phi(t-\xi) \in \mathcal{C}\left([-\bar{\tau}+\xi, \xi], \mathbb{R}^{n}\right)$.

Lemma 2: For the lag-synchronization error system (9), if there exist a positive number $\lambda$ and positive definite diagonal matrices $L=\operatorname{diag}\left\{l_{1}, l_{2}, \ldots, l_{n}\right\}, \digamma=\operatorname{diag}\left(\digamma_{1}, \digamma_{2}, \ldots, \digamma_{n}\right)$, $R=\operatorname{diag}\left(r_{1}, r_{2}, \ldots, r_{n}\right)$, and

$$
\left.\Theta_{l}=-2 \lambda \digamma L^{-1}-\right\urcorner_{l}+R+2\|\digamma\|\left\|B_{l}\right\|_{2} I<0
$$

where $\left.\left.\lambda=\min _{l=1,2, \ldots, N}\left\{\lambda_{\min }\left\{C_{l}\right\}\right\}, \quad\right\rceil_{l}=(\rceil_{l i j}\right)_{n \times n}$ with \rceil$_{l i i}=-2 \digamma_{i} \hat{a}_{l i i}$, and $\rceil_{l i j}=-\left(\digamma_{i} \hat{a}_{l i j}+\digamma_{j} \hat{a}_{l j i}\right)$, for $i \neq j$, such that $\left.\dot{V}(t)\right|_{(9)} \leq-\beta \lambda / 2 e^{T}(t) e(t)$, where

$$
\begin{aligned}
V(t)=\frac{\beta}{2} e^{T}(t) e(t)+ & 2 \sum_{i=1}^{n} \digamma_{i} \int_{0}^{e_{i}(t)} \Phi_{i}(s) d s \\
& +\sum_{i=1}^{n} \int_{t-\tau_{i}(t)}^{t} \Phi_{i}^{2}\left(e_{i}(s)\right) r_{i} d s
\end{aligned}
$$

Proof: Let $V_{1}(t)=1 / 2 e^{T}(t) e(t), \quad V_{2}(t)=$ $2 \sum_{i=1}^{n} \digamma_{i} \int_{0}^{e_{i}(t)} \Phi_{i}(s) d s+\sum_{i=1}^{n} \int_{t-\tau_{i}(t)}^{t} \Phi_{i}^{2}\left(e_{i}(s)\right) r_{i} d s$. Then

$$
V(t)=\beta V_{1}(t)+V_{2}(t)
$$

where the scalar $\beta>0$. Then

$$
\dot{V}(t)=\beta \dot{V}_{1}(t)+\dot{V}_{2}(t)
$$

where

$$
\begin{aligned}
\dot{V}_{1}(t)= & \sum_{l=1}^{N} \Pi_{l}(t)\left(-e^{T}(t) C_{l} e(t)+e^{T}(t) \hat{A}_{l} \Phi(e(t))\right. \\
& \left.+e^{T}(t) B_{l} \Phi(e(t-\tau(t)))\right) \\
\dot{V}_{2}(t)= & 2 \sum_{i=1}^{n} \digamma_{i} \Phi_{i}^{T} \dot{e}_{i}(t) \\
& +\sum_{i=1}^{n} r_{i}\left(\Phi_{i}^{T}\left(e_{i}(t)\right) \Phi_{i}\left(e_{i}(t)\right)-(1-\dot{\tau}(t)) \Phi_{i}^{T}\right. \\
& \left.-\left(e_{i}(t-\tau(t))\right) \Phi_{i}\left(e_{i}(t-\tau(t))\right)\right) \\
= & 2 \Phi^{T}(e(t)) \digamma \dot{e}(t)+\Phi^{T}(e(t)) R \Phi(e(t)) \\
= & 2 \sum_{l=1}^{N} \Pi_{l}(t)\left(-\Phi^{T}(e(t)) \digamma C_{l} e(t)\right. \\
& +(1-\dot{\tau}(t)) \Phi^{T}(e(t-\tau(t))) R \Phi(e(t-\tau(t))) . \\
& +2 \Phi^{T}(e(t)) \digamma \hat{A}_{l} \Phi(e(t)) \\
& \left.+2 \Phi^{T}(e(t)) \digamma B_{l} \Phi(e(t-\tau(t)))\right) \\
&
\end{aligned}
$$

As $\dot{V}_{1}(t)$ can be presented as

$$
\begin{aligned}
\dot{V}_{1}(t)=\sum_{l=1}^{N} \Pi_{l}(t)( & -e^{T}(t) C_{l} e(t)+\left(e^{T}(t) \frac{\left(C_{l}\right)^{\frac{1}{2}}}{\sqrt{2}}\right) \\
& \times \sqrt{2}\left(C_{l}\right)^{\frac{-1}{2}} \hat{A}_{l} \Phi(e(t))+\left(e^{T}(t) \frac{\left(C_{l}\right)^{\frac{1}{2}}}{\sqrt{2}}\right) \\
& \left.\times \sqrt{2}\left(C_{l}\right)^{\frac{-1}{2}} B \Phi(e(t-\tau(t)))\right) .
\end{aligned}
$$


By Lemma 1

$$
\begin{aligned}
& \dot{V}_{1}(t) \\
& \leq \frac{1}{2} \sum_{l=1}^{N} \Pi_{l}(t)\left(-e^{T} C_{l} e(t)+2 \Phi^{T}(e(t)) \hat{A}_{l}^{T} C_{l}^{-1} \hat{A}_{l} \Phi(e(t))\right. \\
& \left.+2 \Phi^{T}(e(t-\tau(t))) B_{l}^{T} C_{l}^{-1} B_{l} \Phi(e(t-\tau(t)))\right) \\
& \leq \frac{1}{2} \sum_{l=1}^{N} \Pi_{l}(t)\left(-e^{T}(t) C_{l} e(t)+2\left\|C_{l}^{-1}\right\|\left\|\hat{A}_{l}\right\|_{2}^{2}\right. \\
& \times \Phi^{T}(e(t)) \Phi(e(t))+2\left\|C _ { l } ^ { - 1 } \left|\left\||| B_{l}\right\|_{2}^{2}\right.\right. \\
& \left.\times \Phi^{T}(e(t-\tau(t))) \Phi(e(t-\tau(t)))\right) \\
& \leq-\frac{\lambda}{2} \sum_{l=1}^{N} \Pi_{l}(t) e^{T}(t) e(t) \\
& +\sum_{l=1}^{N} \Pi_{l}(t)\left(\left\|C_{l}^{-1}\left|\|\| \hat{A}_{l}\left\|_{2}^{2} \Phi^{T}(e(t)) \Phi(e(t))+\right\| C_{l}^{-1}\right|\right\||| B_{l} \|_{2}^{2}\right. \\
& \left.\times \Phi^{T}(e(t-\tau(t))) \Phi(e(t-\tau(t)))\right) \\
& \leq-\frac{\lambda}{2} e^{T}(t) e(t)+M \Phi^{T}(e(t)) \Phi(e(t)) \\
& +M \Phi^{T}(e(t-\tau(t))) \Phi(e(t-\tau(t)))
\end{aligned}
$$

where

$$
M=\max _{l=1, \ldots, N}\left\{\lambda^{-1}\left\|\hat{A}_{l}\right\|_{2}^{2}, \lambda^{-1}\left\|B_{l}\right\|_{2}^{2}\right\} \geq 0
$$

As $e_{i}(t) \Phi_{i}(t) \geq l_{i}^{-1}\left(\Phi_{i}(t)\right)^{2}$, we have

$$
\begin{aligned}
& -2 \Phi^{T}(e(t)) \digamma \sum_{l=1}^{N} \Pi_{l}(t) C_{l} e(t) \\
& \leq-2 \Phi^{T}(e(t)) \digamma \sum_{l=1}^{N} \Pi_{l}(t) C_{l} L^{-1} \Phi(e(t)) .
\end{aligned}
$$

Therefore

$$
\begin{aligned}
\dot{V}_{2}(t) \leq & 2 \Phi^{T}(e(t)) \digamma \\
& \times \sum_{l=1}^{N} \Pi_{l}(t)\left(-C_{l} L^{-1} \Phi(e(t))\right. \\
& +2 \Phi^{T}(e(t)) \digamma \hat{A}_{l} \Phi(e(t)) \\
& \left.+2 \Phi^{T}(e(t)) \digamma B_{l} \Phi(e(t-\tau(t)))\right) \\
& +\Phi^{T}(e(t)) R \Phi(e(t)) \\
& -(1-\mu) \Phi^{T}(e(t-\tau(t))) R \Phi(e(t-\tau(t))) \\
\leq & -\Phi^{T}(e(t))\left(2 \lambda \digamma L^{-1}\right) \Phi(e(t)) \\
& -\sum_{l=1}^{N} \Pi_{l}(t)\left(\Phi^{T}(e(t))\right\rceil_{l} \Phi(e(t))-2\|\digamma\|\left\|B_{l}\right\|_{2} \\
& \times \Phi^{T}(e(t)) R \Phi(e(t)) \\
& \left.-(1-\mu) \Phi^{T}(e(t-\tau(t))) R \Phi(e(t-\tau(t))) . \quad(11)\|||(e(t-\tau(t)))\|\right)
\end{aligned}
$$

Furthermore, we can obtain

$$
\begin{aligned}
& 2\|\digamma\|\left\|B_{l}\right\|_{2}\left\|\Phi^{T}(e(t))\right\|\|\Phi(e(t-\tau(t)))\| \\
& \leq\|\digamma\|\left\|B_{l}\right\|_{2}\left(\Phi^{T}(e(t)) \Phi(e(t))\right. \\
& \left.\quad+\Phi^{T}(e(t-\tau(t))) \Phi(e(t-\tau(t)))\right) .
\end{aligned}
$$

From (11) and (12), we have

$$
\begin{aligned}
\dot{V}_{2}(t) & \\
\leq \sum_{l=1}^{N} \Pi_{l}(t)( & \left.\Phi^{T}(e(t))\left(-2 \lambda \digamma L^{-1}-\right\rceil_{l}+R+\|\digamma\|\left\|B_{l}\right\|_{2}\right) \\
& \times \Phi(e(t))-\Phi^{T}(e(t-\tau(t)))((1-\mu) R \\
& \left.\left.-\|\digamma\|\left\|B_{l}\right\|_{2}\right) \Phi(e(t-\tau(t)))\right) .
\end{aligned}
$$

As $\Theta_{l}<0$, there exists $v>0$ such that $\Theta_{l}+2 v I<0$. Define

$$
R=\max _{l=1, \ldots, N}\left\{\frac{1}{1-\mu}\left(\|\digamma\|\left\|B_{l}\right\|_{2}+v\right)\right\} I>0 .
$$

Then, we can obtain

$\dot{V}_{2}(t) \leq-v \Phi^{T}(e(t)) \Phi(e(t))-v \Phi^{T}(e(t-\tau(t))) \Phi(e(t-\tau(t)))$.

Let

$$
\beta= \begin{cases}\frac{v}{\varpi M}, & M>0 \\ 1, & M=0\end{cases}
$$

where $\varpi \geq 1$. Then, $\dot{V}(t) \leq-\beta \lambda / 2 e^{T}(t) e(t)$.

Moreover, we can obtain the following theorem.

Theorem 1: Assume that the conditions in Lemma 2 hold, then the driving system (4) is globally exponentially lag synchronized with the response system (5).

Proof: Let $\lambda=\min _{l=1, \ldots, N}\left\{\lambda_{\min }\left\{C_{l}\right\}\right\}$. As $V(t)$ defined in Lemma 2 is a positive definite and radially unbounded Lyapunov functional. We can choose a positive number $\epsilon>0$ to satisfy

$$
\epsilon \beta-\lambda \beta+2 \epsilon\|L \digamma\|+2 \epsilon \bar{\tau} e^{\epsilon \bar{\tau}}\left\|L^{2} R\right\|<0 .
$$

By Lemma 2, we can obtain

$$
\begin{aligned}
& \frac{d}{d t}\left\{e^{\epsilon t} V(t)\right\} \\
& =e^{\epsilon t}(\epsilon V(t)+\dot{V}(t)) \\
& \leq \sum_{l=1}^{N} \Pi_{l}(t) e^{\epsilon t}\left(\epsilon \left(\frac{\beta}{2} e^{T}(t) e(t)+2 \sum_{i=1}^{n} \digamma_{i} \int_{0}^{e_{i}(t)} \Phi_{i}(s) d s\right.\right. \\
& \left.\left.+\sum_{i=1}^{n} \int_{t-\tau_{i}(t)}^{t} \Phi_{i}^{2}\left(e_{i}(s)\right) r_{i} d s\right)-\frac{\beta \lambda}{2} e^{T}(t) e(t)\right) \\
& \leq \frac{1}{2} \sum_{l=1}^{N} \Pi_{l}(t) e^{\epsilon t}\left(\epsilon \beta e^{T}(t) e(t)-\beta \lambda e^{T}(t) e(t)\right. \\
& \left.+\epsilon \epsilon \sum_{i=1}^{n} \digamma_{i} \int_{0}^{e_{i}(t)} \Phi_{i}(s) d s\right) \\
& \sum_{i=1}^{n} \int_{t-\tau_{i}(t)}^{t} \Phi_{i}^{2}\left(e_{i}(s)\right) r_{i} d s .
\end{aligned}
$$


Since

$$
\begin{aligned}
\sum_{i=1}^{n} \digamma_{i} \int_{0}^{e_{i}(t)} \Phi_{i}(s) d s & \\
& \leq \sum_{i=1}^{n} \digamma_{i} \int_{0}^{e_{i}(t)} l_{i} s d s \leq \frac{1}{2} e^{T}(t) L \digamma e(t)
\end{aligned}
$$

then

$$
\begin{aligned}
& \frac{d}{d t}\left(e^{\epsilon t} V(t)\right) \\
& \leq \frac{1}{2} \sum_{l=1}^{N} \Pi_{l}(t) e^{\epsilon t}\left(\epsilon \beta e^{T}(t) e(t)-\beta \lambda e^{T}(t) e(t)+2 \epsilon e^{T}(t) L \digamma e(t)\right. \\
& \left.+\epsilon e^{\epsilon t} \sum_{i=1}^{n} \int_{t-\tau_{i}(t)}^{t} \Phi_{i}^{2}\left(e_{i}(s)\right) r_{i} d s\right) \\
& \leq \frac{1}{2} e^{\epsilon t}(\epsilon \beta-\lambda \beta+2 \epsilon\|L \digamma\|) e^{T}(t) e(t) \\
& +\epsilon e^{\epsilon t} \sum_{i=1}^{n} \int_{t-\tau_{i}(t)}^{t} \Phi_{i}^{2}\left(e_{i}(s)\right) r_{i} d s .
\end{aligned}
$$

Changing the integral of the second term on the right-hand side of (14)

$$
\begin{aligned}
\epsilon \int_{0}^{s} e^{\epsilon t} \sum_{i=1}^{n} \int_{t-\tau_{i}(t)}^{t} \Phi_{i}^{2}\left(e_{i}(\varsigma)\right) r_{i} d \varsigma d t \\
\quad=\epsilon \sum_{i=1}^{n} \int_{0}^{s} e^{\epsilon t} \int_{t-\tau_{i}(t)}^{t} \Phi_{i}^{2}(\varsigma) r_{i} d \varsigma d t \\
\quad \leq \epsilon \sum_{i=1}^{n} \int_{-\bar{\tau}}^{s} \int_{\max \{\varsigma, 0\}}^{\min \{\varsigma+\bar{\tau}, s\}} e^{\epsilon t} d t \Phi_{i}^{2}\left(e_{i}(\varsigma)\right) r_{i} d \varsigma \\
\quad \leq \epsilon \sum_{i=1}^{n} \int_{-\bar{\tau}}^{s}\left(\int_{\varsigma}^{\varsigma+\bar{\tau}} e^{\epsilon t} d t\right) \Phi_{i}^{2}\left(e_{i}(\varsigma)\right) r_{i} d \varsigma \\
\quad \leq \sum_{i=1}^{n} \int_{-\bar{\tau}}^{s} \bar{\tau} e^{\epsilon(\varsigma+\bar{\tau})} \Phi_{i}^{2}\left(e_{i}(\varsigma)\right) r_{i} d \varsigma \\
\quad \leq \sum_{i=1}^{n} \int_{-\bar{\tau}}^{s} \bar{\tau} e^{\epsilon(\varsigma+\bar{\tau})} e_{i}^{2}(\varsigma) l_{i}^{2} r_{i} d \varsigma \\
\leq \bar{\tau} e^{\epsilon \bar{\tau}}\left\|L^{2} R\right\| \int_{-\bar{\tau}}^{s} e^{\epsilon \varsigma} e^{T}(\varsigma) e(\varsigma) d \varsigma \\
\leq \bar{\tau} e^{\epsilon \bar{\tau}}\left\|L^{2} R\right\|\left(\int_{-\bar{\tau}}^{0} e^{\epsilon \varsigma} e^{T}(\varsigma) e(\varsigma) d \varsigma\right. \\
\left.\quad+\int_{0}^{s} e^{\epsilon \varsigma} e^{T}(\varsigma) e(\varsigma) d \varsigma\right) .
\end{aligned}
$$

By (13)-(15), we have

$$
\begin{aligned}
& e^{\epsilon S} V(s)-V(0) \\
& \leq \frac{1}{2}\left(\epsilon \beta-\lambda \beta+2 \epsilon\|L \digamma\|+2 \epsilon \bar{\tau} e^{\epsilon \bar{\tau}}\left\|L^{2} R\right\|\right) \\
& \times \int_{0}^{s} e^{\epsilon t} e^{T}(t) e(t) d t+\epsilon \bar{\tau} e^{\epsilon \bar{\tau}}\left\|L^{2} R\right\| \int_{-\bar{\tau}}^{0} e^{\epsilon t} e^{T}(t) e(t) d t \\
& \leq\left(\epsilon \bar{\tau}\left\|L^{2} R\right\| \int_{-\bar{\tau}}^{0} e^{\epsilon t} d t\right)\|\psi\|^{2} \equiv H_{1}\|\psi\|^{2} .
\end{aligned}
$$

Thus

$$
V(t) \leq\left(V(0)+H_{1}\|\psi\|^{2}\right) e^{-\epsilon t} \quad \forall t>0
$$

where

$$
\begin{aligned}
V(0)= & \frac{\beta}{2} e^{T}(0) e(0)+2 \sum_{i=1}^{n} q_{i} \int_{0}^{e_{i}(0)} \Phi_{i}(s) d s \\
& +\sum_{i=1}^{n} \int_{-\tau_{i}(t)}^{0} \Phi_{i}^{2}\left(e_{i}(s)\right) r_{i} d s \\
\leq & \frac{1}{2}\left(\beta+2\|\digamma L\|+2 \bar{\tau}\left\|L^{2} R\right\|\right)\|\psi\|^{2} \\
\equiv & H_{2}\|\psi\|^{2} .
\end{aligned}
$$

By (10) and (16), we have

$$
\frac{\beta}{2} e^{T}(t) e(t) \leq V(t) \leq\left(H_{1}+H_{2}\right)\|\psi\|^{2} e^{-\epsilon t} \quad \forall t>0 .
$$

Thus, we have

$$
\|e(t)\| \leq \sqrt{\frac{2}{\beta}\left(H_{1}+H_{2}\right)}\|\psi\| e^{-\frac{\epsilon}{2} t}
$$

which implies the drive system (4) is globally exponentially lag synchronized with the response system (5). This completes the proof.

By Theorem 1, we can obtain the following corollary.

Corollary 1: The drive system (4) is globally exponentially lag synchronized with the response system (5), if there exist positive definite matrices $L=\operatorname{diag}\left\{l_{1}, l_{2}, \ldots, l_{n}\right\}$, $\digamma=\operatorname{diag}\left\{\digamma_{1}, \digamma_{2}, \ldots, \digamma_{n}\right\}, R=\operatorname{diag}\left\{r_{1}, r_{2}, \ldots, r_{n}\right\}$ and a positive definite symmetric matrix $M$, such that

$$
\left.\hat{\Pi}_{l}=-2 \lambda \digamma L^{-1}-\right\rceil_{l}+R+M+\|\digamma\|^{2}\left\|M^{-1}\right\|\left\|B_{l}\right\|_{2}^{2} I<0
$$

where $\left.\left.\lambda=\min _{l=1, \ldots, N}\left\{D_{l}\right\},\right\rceil_{l}=(\urcorner_{l i j}\right)_{n \times n}$ with $\rceil_{l i i}=-2 \digamma_{i} \hat{a}_{l i i}$, and $\urcorner_{l i j}=-\left(\digamma_{i} \hat{a}_{l i j}+\digamma_{j} \hat{a}_{l j i}\right)$, for $i \neq j$.

Proof: By Lemma 1

$$
\frac{1}{\|\digamma\|\left\|B_{l}\right\|_{2}} M+\|\digamma\|\left\|B_{l}\right\|_{2} M^{-1} \geq 2 I
$$

then

$$
\begin{aligned}
M+\|\digamma\|^{2}\left\|M^{-1}\right\|\left\|B_{l}\right\|_{2}^{2} I & \geq M+\|\digamma\|^{2}\left\|B_{l}\right\|_{2}^{2} M^{-1} \\
& \geq 2\|\digamma\|\left\|B_{l}\right\|_{2}^{2} I .
\end{aligned}
$$

Thus

$$
\begin{aligned}
-2 & \lambda \digamma L^{-1}-7+R+2\|\digamma\|\left\|B_{l}\right\|_{2} I \\
& \leq-2 \lambda \digamma L^{-1}-7_{l}+R+M+\|\digamma\|^{2}\left\|M^{-1}\right\|\left\|B_{l}\right\|_{2}^{2} I \\
& <0 .
\end{aligned}
$$

This proof is complete.

Let $\digamma=M=I$ in Corollary 1, we obtain the following corollary.

Corollary 2: The drive system (4) is globally exponentially lag synchronized with the response system (5), if \urcorner$\left._{l}=(\rceil_{l i j}\right)_{n \times n}$ is positive definite, and $\left\|B_{l}\right\|_{2} \leq(2 \pi-1)^{1 / 2}$, where $\pi=\min _{1 \leq i \leq n}\left(\lambda / l_{i}\right)$, and $\rceil_{l i i}=-2 \digamma_{i} \hat{a}_{l i i}$, \urcorner$_{l i j}=-\left(\digamma_{i} \hat{a}_{l i j}+\digamma_{j} \hat{a}_{l j i}\right)$, for $i \neq j$. 

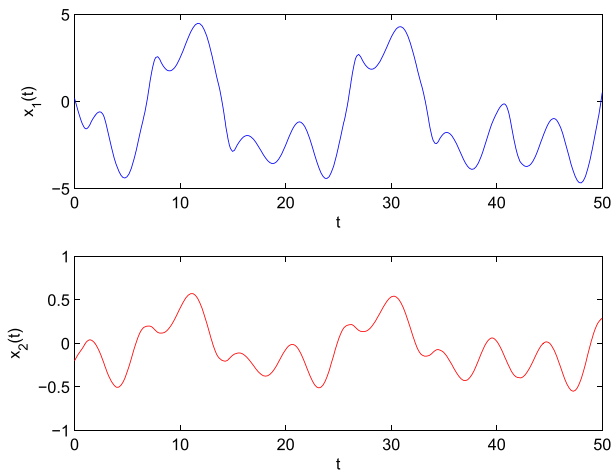

(a)

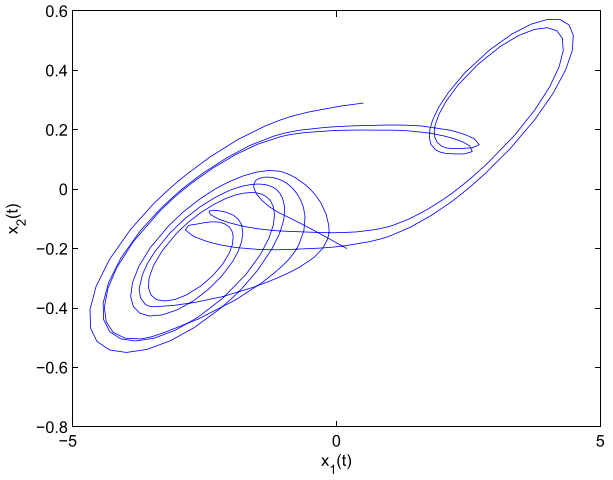

(b)

Fig. 2. Transient behavior of the switched system (4) with the initial value $[0.2-0.2]^{T}$.

Some criteria about globally exponential stability can be derived for the switched lag-synchronization error system (9) with the controller of the neural activation function [26]-[31]. The globally asymptotical stability conditions for for the switched lag-synchronization error system in [26, eq. (9)] and [27, eq. (9)] are presented as follows.

Corollary 3: The drive system (4) is globally exponentially lag synchronized with the response system (5), if \urcorner$\left._{l}=(\urcorner_{l i j}\right)_{n \times n}$ is positive definite, and $\left\|B_{l}\right\|_{2} \leq \pi$, where $\pi=\min _{1 \leq i \leq n}\left(\lambda / l_{i}\right)$, and $\left.\rceil_{l i i}=-2 \digamma_{i} \hat{a}_{l i i},\right\rceil_{l i j}=-\left(\digamma_{i} \hat{a}_{l i j}+\right.$ $\left.\digamma_{j} \hat{a}_{l j i}\right)$, for $i \neq j$.

Corollary 4: The synchronization error system (9) is globally asymptotically stable if there exist a positive definite diagonal matrices $\digamma=\operatorname{diag}\left\{\digamma_{1}, \digamma_{2}, \ldots, \digamma_{n}\right\}$, $R=\operatorname{diag}\left\{r_{1}, r_{2}, \ldots, r_{n}\right\}$ such that

$$
\left.\hat{\Pi}_{l}=-2 \omega I-\right\urcorner_{l}+R+2\|\digamma\|^{2}\left\|B_{l}\right\|_{2}^{2} I<0
$$

where $\left.\left.\omega=\min _{1 \leq i \leq n}\left(\digamma_{i} \lambda / l_{i}\right), \quad\right\urcorner_{l}=(\urcorner_{l i j}\right)_{n \times n}$ with \rceil$\left._{l i i}=-2 \digamma_{i} \hat{a}_{l i i},\right\rceil_{l i j}=-\left(\digamma_{i} \hat{a}_{l i j}+\digamma_{j} \hat{a}_{l j i}\right)$, for $i \neq j$.

The following inequality holds:

$$
\begin{aligned}
\tilde{\Pi}_{l} & =-2 \omega I-\urcorner_{l}+R+2\|\digamma\|^{2}\left\|B_{l}\right\|_{2}^{2} I \\
& \left.\geq-2 \lambda \digamma L^{-1}-\right\urcorner_{l}+R+\|\digamma\|^{2}\left\|B_{l}\right\|_{2}^{2} I
\end{aligned}
$$

where $\omega=\min _{1 \leq i \leq n}\left(\digamma_{i} \lambda / l_{i}\right)$. This means the conditions about stability criteria derived for switched neural networks in [26] and [27] are more restrictive than those in Theorem 1. Meanwhile, these results can only guarantee the globally
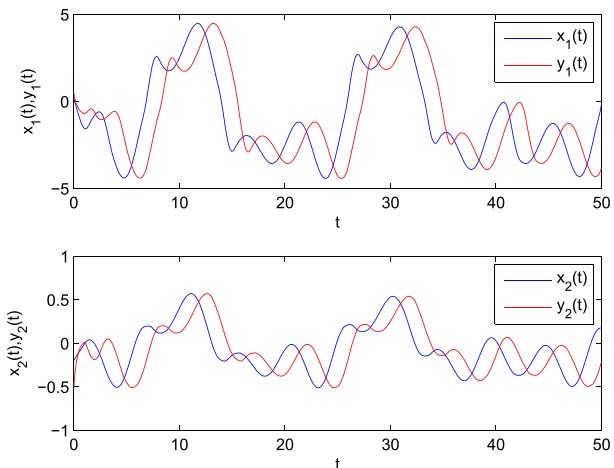

(a)

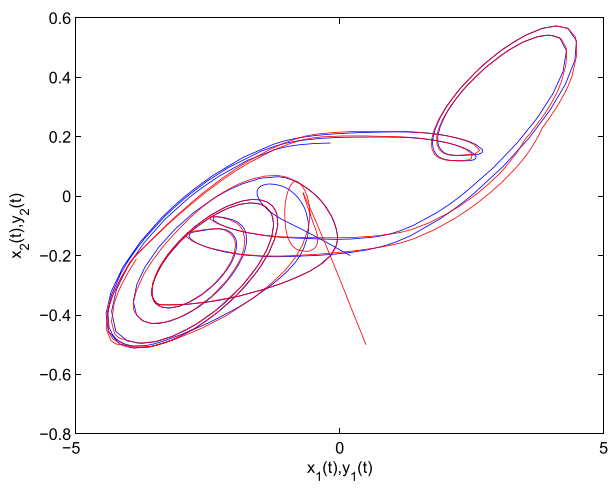

(b)

Fig. 3. (a) and (b) State trajectories of driving system (4) with the initial value $[0.2-0.2]^{T}$ and slave system (5) with the initial value $[0.5-0.5]^{T}$, when lag time $\xi=1.5$.

asymptotical stability of the switched lag synchronization error system (9).

\section{ILLUSTRATIVE EXAMPLES}

To show the effectiveness of the obtained results, two illustrative examples are presented as follows.

Example 1: Consider a switched system (4) with

$$
\begin{aligned}
A & =\left[\begin{array}{ll}
1.8 & 10 \\
0.1 & 1.8
\end{array}\right], \quad B=\left[\begin{array}{ll}
-1.5 & 0.1 \\
0.1 & -1.5
\end{array}\right] \\
f_{i}\left(x_{i}\right) & =\frac{1}{2}\left(\left|x_{i}+1\right|-\left|x_{i}-1\right|\right) \\
\tau_{i}(t) & =0.97, i=1,2 .
\end{aligned}
$$

If $x_{1}(t) \leq 0$

$$
C=\left[\begin{array}{ll}
1.0 & 0 \\
0 & 1.2
\end{array}\right]
$$

Else

$$
C=\left[\begin{array}{ll}
1.2 & 0 \\
0 & 1.0
\end{array}\right]
$$

The initial values of driving system (4) is set to be $[0.2-0.2]^{T}$. In addition, the dynamical behaviors of this system is shown as in Fig. 2, which is chaotic and can be used in secure communications.

As

$$
\lambda=\min _{l=1,2}\left\{\lambda_{\min }\left\{C_{l}\right\}\right\}=1
$$



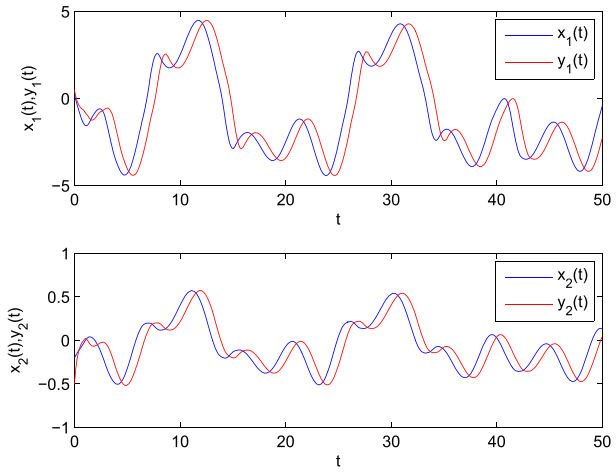

(a)

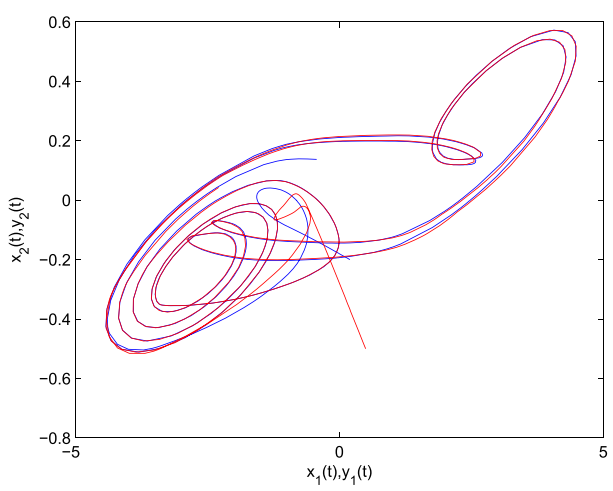

(b)

Fig. 4. (a) and (b) State trajectories of driving system (4) with the initial value $[0.2-0.2]^{T}$ and slave system (5) with the initial value $[0.5-0.5]^{T}$, when lag time $\xi=0.8$.

Obviously, there exists a positive definite diagonal matrix $\digamma=\operatorname{diag}\{0.5,0.5\}, v=0.1, R=1 /(1-\mu)\{(\|\digamma\|$ $\left.\left.\|B\|_{2}+v\right) I\right\}$, such that

$$
\rceil_{l}=-\hat{A}_{l}
$$

to make

$$
\begin{aligned}
\Theta_{l} & \left.=-2 \lambda \digamma L^{-1}-\right\rceil_{l}+R+\|\digamma\|\left\|B_{l}\right\|_{2} I \\
& =K_{l}+\left[\begin{array}{ll}
3.3 & 11 \\
1 & 3.3
\end{array}\right]<0
\end{aligned}
$$

therefore

$$
K_{l}<\left[\begin{array}{ll}
-3.3 & -11 \\
-1 & -3.3
\end{array}\right]
$$

can make the lag-synchronization error system (9) globally exponentially stable. However, $\|B\|_{2}=1.6>\varpi$, which means that the results in [26] cannot be used for the lag-synchronization error system (9). To simulate the obtained result, let

$$
K_{l}=\left[\begin{array}{ll}
-3.6 & -11 \\
-1 & -3.6
\end{array}\right] .
$$

Set the initial states of slave system (9) is $[0.5-0.5]^{T}$. The state trajectories of driving system and slave system are presented in Figs. 3-5 with the lag times $\xi=1.5, \xi=0.8$, and $\xi=0$, respectively, which illustrate the effectiveness of the obtained results.
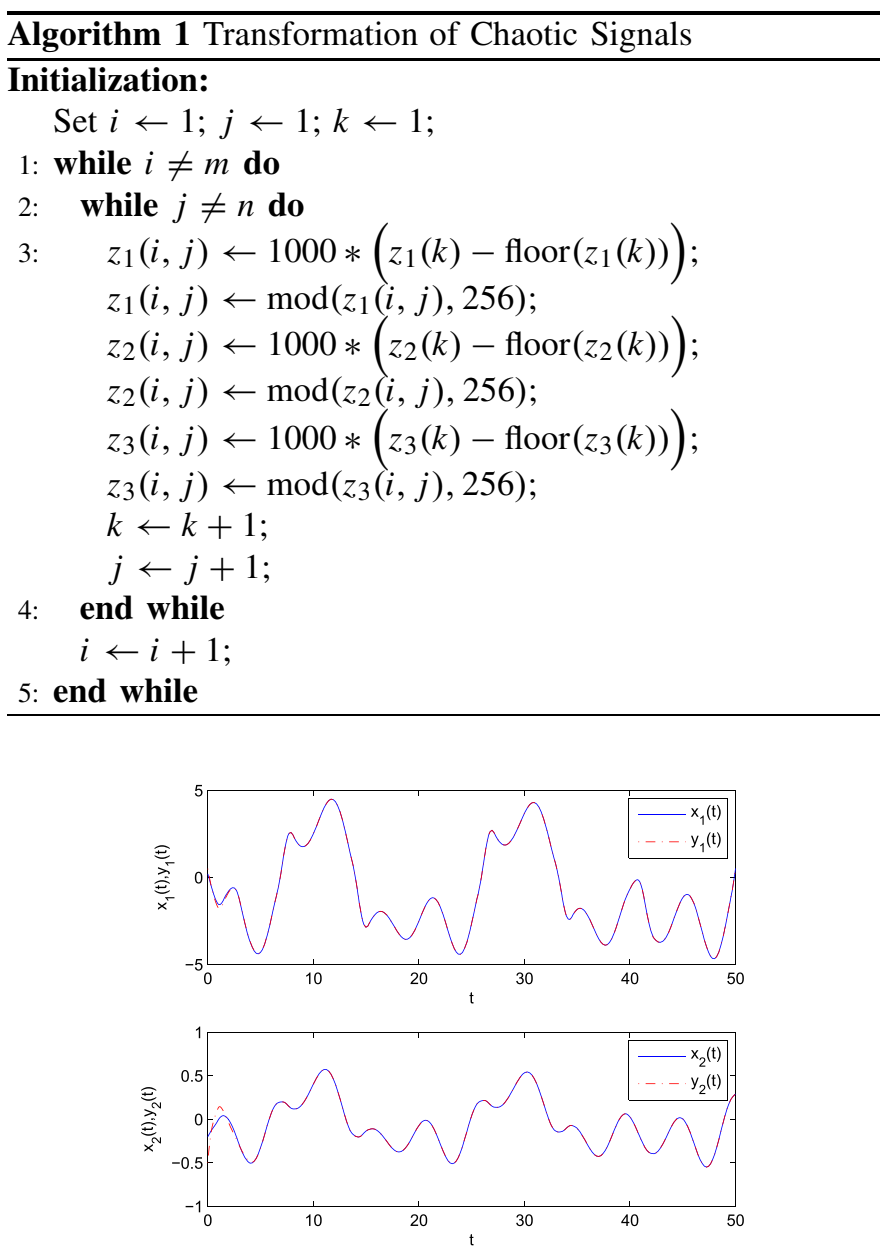

(a)

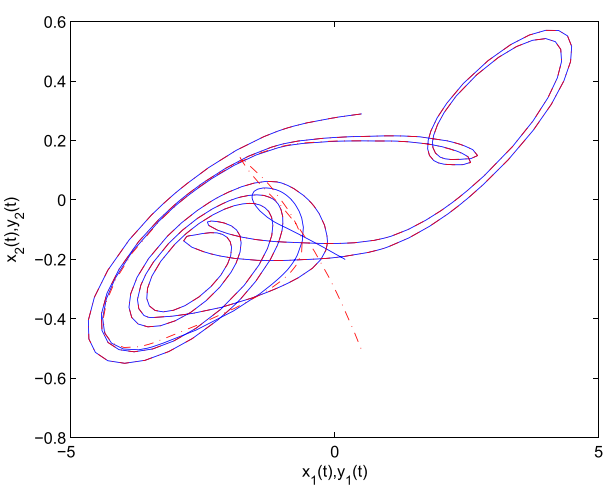

(b)

Fig. 5. (a) and (b) State trajectories of driving system (4) with the initial value $[0.2-0.2]^{T}$ and slave system (5) with the initial value $[0.5-0.5]^{T}$, when lag time $\xi=0$.

Example 2: Based on Example 1, the obtained results can be applied in the field of digital signal processing, and the algorithm is presented for a color picture $F$ with a size $m \times n \times 3$, as follows.

1) Separating color image $F$ into three gray ones with red, green, and blue, respectively, and via sort function to rearrange the pixels in each gray image, 


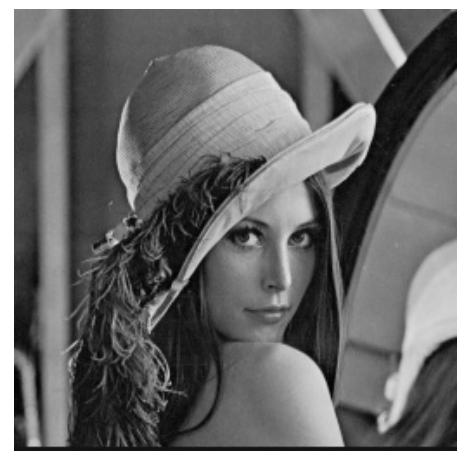

(a)

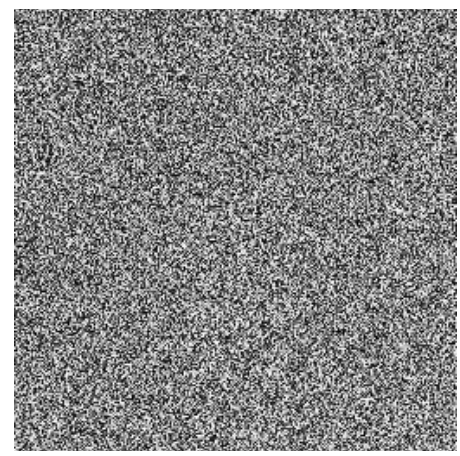

(b)

Fig. 6. (a) Original image. (b) Encrypted image.

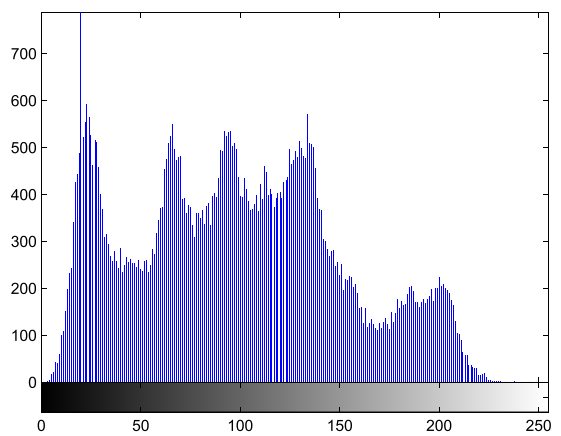

(a)

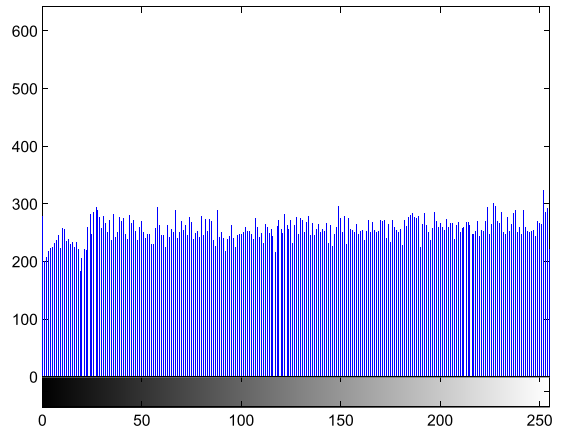

(b)

Fig. 7. (a) Histogram of the original gray image. (b) Histogram of the encrypted gray image.

therefore, three new ordered pixel series are obtained as $R(i, j), G(i, j), B(i, j), i \in\{1, \ldots, m\}, j \in\{1, \ldots, n\}$.

2) Through driving system (4), two groups of timeseries chaotic signals obtained as $z_{l}(i, j)=x_{l}(k)$,

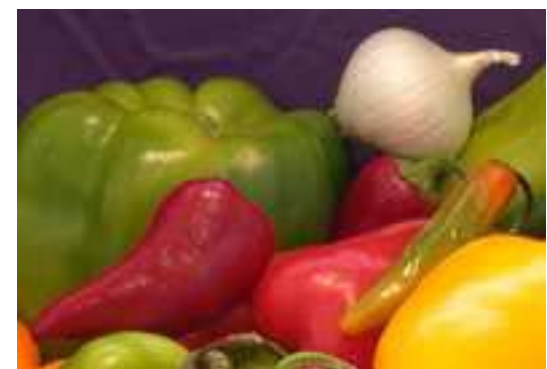

(a)

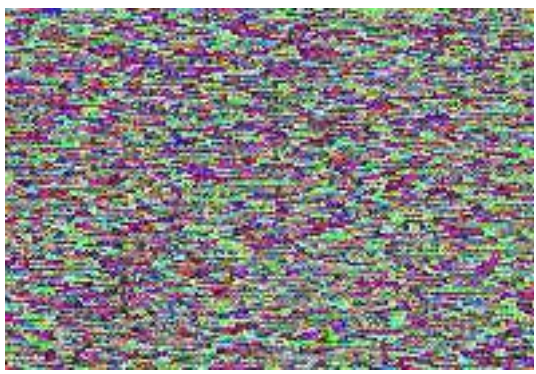

(b)

Fig. 8. (a) Original image. (b) Encrypted image.

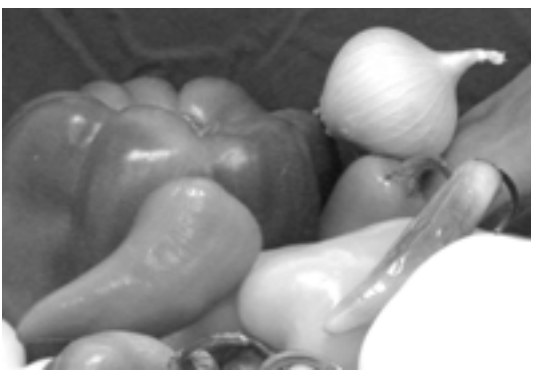

(a)

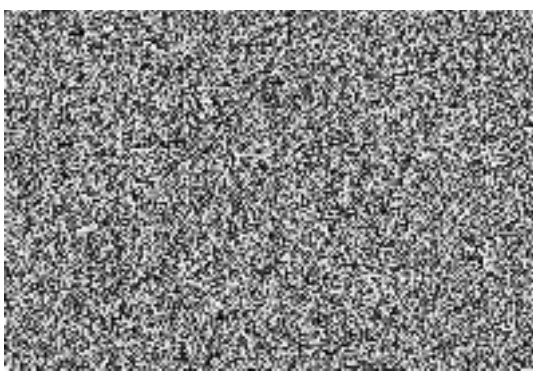

(b)

Fig. 9. (a) Gray image of the red channel of the original image. (b) Same plots for the encrypted image.

$k \in\{1, \ldots, m n\}, l \in\{1,2\}$. As there are three gray images needed to be encrypted, the third chaotic signal can be set as $z_{3}(k)=0.5\left(x_{1}(k)+x_{2}(k)\right)$. After certain transformation, the chaotic signals can be presented as in Algorithm 1.

3) Based on the proceeded chaotic signals and gray images, the encrypted gray images can be obtained as the 


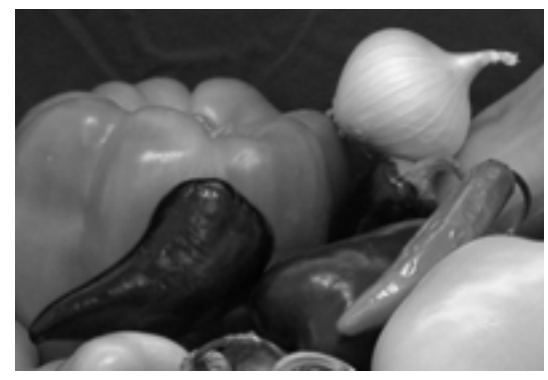

(a)

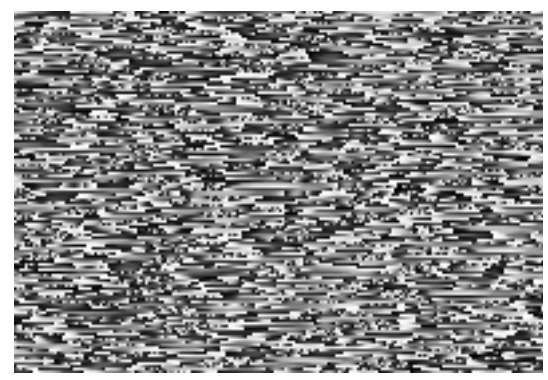

(b)

Fig. 10. (a) Gray image of the green channel of the original image. (b) Same plots for the encrypted image.

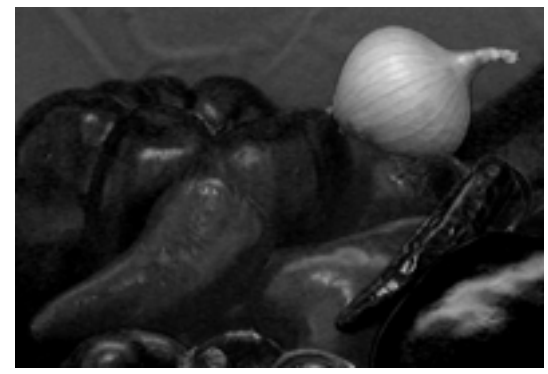

(a)

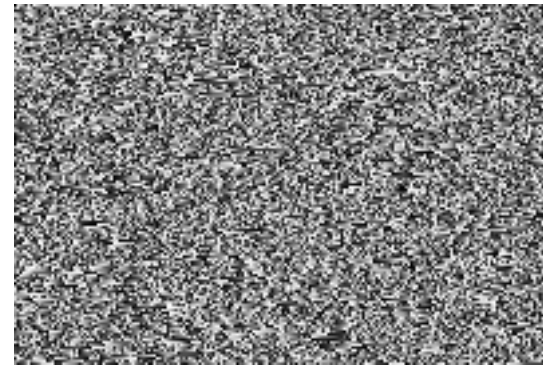

(b)

Fig. 11. (a) Gray image of the blue channel of the original image. (b) Same plots for the encrypted image.

following operation:

$$
\begin{aligned}
& R(i, j) \leftarrow \bmod (R(i, j) * 1000,256) \oplus z_{1}(i, j) \\
& G(i, j) \leftarrow \bmod (G(i, j) * 1000,256) \oplus z_{2}(i, j) \\
& B(i, j) \leftarrow \bmod (G(i, j) * 1000,256) \oplus z_{3}(i, j) .
\end{aligned}
$$

4) Reorganizing $R(i, j), G(i, j)$, and $B(i, j)$, we can obtain the encrypted color image.

As the decryption process is the same as the encryption process, is omitted, we notice that as the existence of lag, the decryption chaotic signal should be employed after $\xi / h$ signals, where $h$ is the length of the iterative step. In addition, two simulations about gray and color image encryption have been provided in Figs. 6-11, which illustrate the application potential of the lag synchronization of switched neural networks in signal encryption. As the existence of the lag between the coupled switched neural networks, decryption chaotic signal should be adopted after certain iterative steps.

\section{CONCLUSION}

As the applications of switched neural networks become more and more popular, lag synchronization of such networks becomes necessary. On the other hand, after the switched neural networks have been packed, it is very hard to measure their inner states. Therefore, the authors investigate the problem of global exponential lag synchronization of a class of switched neural networks with time-varying delays via the neural activation controller. Two numerical examples were provided to demonstrate the effectiveness and improvement of the obtained results in this paper.

\section{REFERENCES}

[1] H. Huang, Y. Qu, and H.-X. Li, "Robust stability analysis of switched Hopfield neural networks with time-varying delay under uncertainty," Phys. Lett. A, vol. 345, nos. 4-6, pp. 345-354, 2005.

[2] Z.-G. Wu, P. Shi, H. Su, and J. Chu, "Sampled-data synchronization of chaotic Lur'e systems with time delays," IEEE Trans. Neural Netw. Learn. Syst., vol. 24, no. 3, pp. 410-421, Mar. 2013.

[3] Y. Zhang, D. Guo, and Z. Li, "Common nature of learning between backpropagation and Hopfield-type neural networks for generalized matrix inversion with simplified models," IEEE Trans. Neural Netw. Learn. Syst., vol. 24, no. 4, pp. 579-592, Apr. 2013.

[4] H. Zhang, T. Ma, G.-B. Huang, and C. Wang, "Robust global exponential synchronization of uncertain chaotic delayed neural networks via dualstage impulsive control," IEEE Trans. Syst., Man, Cybern. B, Cybern., vol. 40, no. 3, pp. 831-844, Jun. 2010.

[5] S. Wen, Z. Zeng, T. Huang, and Y. Zhang, "Exponential adaptive lag synchronization of memristive neural networks via fuzzy method and applications in pseudorandom number generators," IEEE Trans. Fuzzy Syst., vol. 22, no. 6, pp. 1704-1713, Dec. 2014.

[6] S. Wen, G. Bao, Z. Zeng, Y. Chen, and T. Huang, "Global exponential synchronization of memristor-based recurrent neural networks with timevarying delays," Neural Netw., vol. 48, pp. 195-203, Dec. 2013.

[7] L. O. Chua and T. Roska, Cellular Neural Networks and Visual Computing: Foundations and Applications. Cambridge, U.K. Cambridge Univ. Press, 2002.

[8] G. Chen and X. Dong, From Chaos to Order: Methodologies, Perspectives and Applications. Singapore: World Scientific, 1998.

[9] L. Wu, Z. Feng, and J. Lam, "Stability and synchronization of discretetime neural networks with switching parameters and time-varying delays," IEEE Trans. Neural Netw. Learn. Syst., vol. 24, no. 12, pp. 1957-1972, Dec. 2013.

[10] H. Zhang, L. Cui, and Y. Luo, "Near-optimal control for nonzero-sum differential games of continuous-time nonlinear systems using singlenetwork ADP," IEEE Trans. Cybern., vol. 43, no. 1, pp. 206-216, Feb. 2013.

[11] H. Zhang, D. Liu, Y. Luo, and D. Wang, Adaptive Dynamic Programming for Control: Algorithms and Stability. London, U.K.: Springer-Verlag, 2013. 
[12] J. Huang, C. Li, T. Huang, and X. He, "Finite-time lag synchronization of delayed neural networks," Neurocomputing, vol. 139, pp. 145-149, Sep. 2014.

[13] Y. Luo, Q. Sun, H. Zhang, and L. Cui, "Adaptive critic design-based robust neural network control for nonlinear distributed parameter systems with unknown dynamics," Neurocomputing, vol. 148, pp. 200-208, Jan. 2015.

[14] H. Zhang, L. Cui, X. Zhang, and Y. Luo, "Data-driven robust approximate optimal tracking control for unknown general nonlinear systems using adaptive dynamic programming method," IEEE Trans. Neural Netw., vol. 22, no. 12, pp. 2226-2236, Dec. 2010.

[15] Y. Xia, Z. Yang, and M. Han, "Synchronization schemes for coupled identical Yang-Yang type fuzzy cellular neural networks," Commun. Nonlinear Sci. Numer. Simul., vol. 14, nos. 9-10, pp. 3645-3659, 2009.

[16] Q. Song, "Design of controller on synchronization of chaotic neural networks with mixed time-varying delays," Neurocomputing, vol. 72, nos. $13-15$, pp. 3288-3295, 2009.

[17] Q. Zhu and J. Cao, "Adaptive synchronization under almost every initial data for stochastic neural networks with time-varying delays and distributed delays," Commun. Nonlinear Sci. Numer. Simul., vol. 16, no. 4, pp. 2139-2159, 2011.

[18] M. Sun, C. Zeng, and L. Tian, "Linear generalized synchronization between two complex networks," Commun. Nonlinear Sci. Numer. Simul., vol. 15, no. 8, pp. 2162-2167, 2010.

[19] F. A. Breve, L. Zhao, M. G. Quiles, and E. E. N. Macau, "Chaotic phase synchronization and desynchronization in an oscillator network for object selection," Neural Netw., vol. 22, nos. 5-6, pp. 728-737, 2009.

[20] W. Yu and J. Cao, "Adaptive Q-S (lag, anticipated, and complete) time-varying synchronization and parameters identification of uncertain delayed neural networks," Chaos, vol. 16, no. 2, pp. 023119-1-023119-11, 2006.

[21] S. Dan, S. X. Yang, and W. Feng, "Lag synchronization of coupled delayed chaotic neural networks by periodically intermittent control," Abstract Appl. Anal., vol. 2013, pp. 501461-1-501461-5, 2013.

[22] J. Huang, C. Li, T. Huang, H. Li, and M. Peng, "Projective lag synchronization of delayed neural networks using intermittent linear state feedback," Abstract Appl. Anal., vol. 2013, pp. 503659-1-503659-5, 2013.

[23] C. Fang, M. Sun, D. Li, and D. Han, "Asymptotically synchronization of a class of chaotic delayed neural networks with impulsive effects via intermittent control," Int. J. Nonlinear Sci., vol. 15, no. 3, pp. 220-227, 2013.

[24] X. Li and M. Bohnerb, "Exponential synchronization of chaotic neural networks with mixed delays and impulsive effects via output coupling with delay feedback," Math. Comput. Model., vol. 52, nos. 5-6, pp. 643-653, 2010.

[25] W. Zhao and Y. Tan, "Harmless delays for global exponential stability of Cohen-Grossberg neural networks," Math. Comput. Simul., vol. 74, no. 1, pp. 47-57, 2007.

[26] S. Arik, "Global robust stability analysis of neural networks with discrete time delays," Chaos Solitons Fractals, vol. 26, no. 5, pp. 1407-1414, 2005.

[27] N. Ozcan and S. Arik, "Global robust stability analysis of neural networks with multiple time delays," IEEE Trans. Circuits Syst. I, Reg. Papers, vol. 53, no. 1, pp. 166-176, Jan. 2006.

[28] S. Wen and Z. Zeng, "Dynamics analysis of a class of memristorbased recurrent networks with time-varying delays in the presence of strong external stimuli," Neural Process. Lett., vol. 35, no. 1, pp. 47-59, 2012.

[29] S. Wen, Z. Zeng, and T. Huang, "Dynamic behaviors of memristor-based delayed recurrent networks," Neural Comput. Appl., vol. 23, nos. 3-4, pp. 815-821, 2013.

[30] X. He, C. Li, T. Huang, and C. Li, "Codimension two bifurcation in a delayed neural network with unidirectional coupling," Nonlinear Anal., Real World Appl., vol. 14, no. 2, pp. 1191-1202, 2013.

[31] X. He, C. Li, T. Huang, C. Li, and J. Huang, "A recurrent neural network for solving bilevel linear programming problem," IEEE Trans. Neural Netw. Learn. Syst., vol. 25, no. 4, pp. 824-830, Apr. 2014.

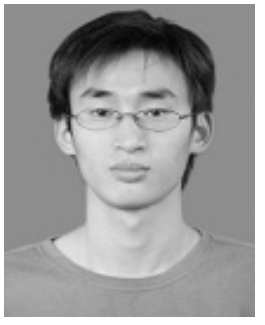

Shiping Wen received the M.S. degree in control science and engineering from the School of Automation, Wuhan University of Technology, Wuhan, China, in 2010, and the Ph.D degree in control science and engineering from the School of Automation, Huazhong University of Science and Technology, Wuhan, China, in 2013.

$\mathrm{He}$ is currently with the School of Automation, Huazhong University of Science and Technology, and the Key Laboratory of Image Processing and Intelligent Control, Ministry of Education, Wuhan. His current research interests include design and analysis of memristor-based circuits and systems, and $\mathrm{H}_{\infty}$ control of networked systems.

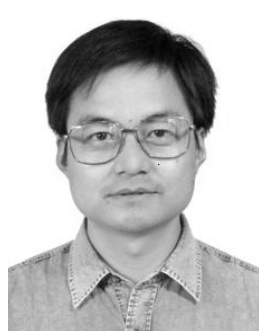

Zhigang Zeng received the B.S. degree from Hubei Normal University, Huangshi, China, in 1993, the M.S. degree from Hubei University, Wuhan, China, in 1996, and the Ph.D. degree from the Huazhong University of Science and Technology, Wuhan, in 2003.

$\mathrm{He}$ is currently a Professor with the School of Automation, Huazhong University of Science and Technology, and also with the Key Laboratory of Image Processing and Intelligent Control, Ministry of Education, Wuhan. His current research interests include neural networks, switched systems, computational intelligence, stability analysis of dynamic systems, pattern recognition, and associative memories.

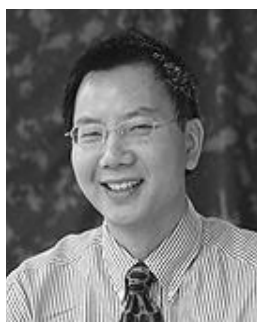

Tingwen Huang received the B.S. degree from Southwest University, Chongqing, China, in 1990, the M.S. degree from Sichuan University, Chengdu, China, in 1993, and the Ph.D. degree from Texas A\&M University, College Station, TX, USA, in 2002.

$\mathrm{He}$ is currently a Professor of Mathematics with Texas A\&M University at Qatar, Doha, Qatar. His current research interests include dynamical systems, memristor, neural networks, complex networks, optimization and control, and traveling wave

phenomena.

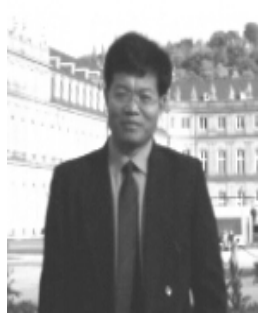

Qinggang Meng is currently a Senior Lecturer with the Department of Computer Science, Research School of Informatics, Loughborough University, Leicester, U.K. He is involved in biologically and psychologically inspired learning algorithms, service robotics, robot learning and adaptation, multiUAV cooperation, human motion analysis and activity recognition, ambient assisted living, driver's distraction detection, and computer vision.

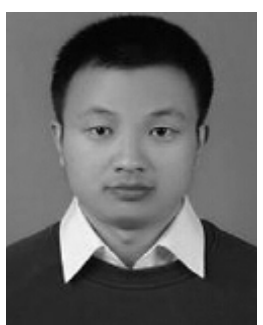

Wei Yao received the B.S. degree in automation from Wuhan University, Wuhan, China, in 2005, and the $\mathrm{Ph} . \mathrm{D}$. degree in control theory and control engineering from the Dalian University of Technology, Dalian, China.

$\mathrm{He}$ is currently a Lecturer with the School of Computer Science, South-Central University for Nationalities, Wuhan. His current research interests include machine learning, neural networks, and theory of forecasting. 